\title{
COMPACT SETS OF FUNCTIONS AND FUNCTION RINGS
}

\section{DAVID GALE}

A widely used theorem of analysis asserts that a uniformly bounded, equicontinuous family of functions has a compact closure in the space of continuous functions. This lemma, variously attributed to Arzela, Escoli, Montel, Vitali, and so on, is of importance in the theory of integral equations, conformal mapping, calculus of variations, and so on. In recent years the lemma has been generalized by S. B. Myers [1]. ${ }^{1}$ A part of his results may be formulated as follows;

If a topological space $X$ is either (a) locally compact, (b) satisfies the first axiom of countability, and if $Y$ is a metric space, then a family $F$ of continuous functions from $X$ to $Y$ is compact (in a suitable topology) if and only if (1) $F(x)=U_{f \in R f} f(x)$ is compact for all $x \in X$, (2) $F$ is closed, (3) $F$ is equicontinuous.

The main purpose of $\S 1$ of this paper is to characterize compact sets of functions when $Y$ is any regular topological space. The problem is therefore to find a condition to replace equicontinuity, which no longer makes sense. We obtain such a characterization which holds for an easily described class of spaces $X$ which includes both locally compact and first countable. In $\$ 2$ these results are applied to obtain a sort of duality theorem for the ring of real-valued continuous functions, $R(X)$, on a space $X$. Namely, it is shown that, under quite general conditions, the space $X$ is homeomorphic with the space $H(X)$ of continuous homomorphisms from the ring $R(X)$ onto the real numbers, where $R(X)$ and $H(X)$ are given the "compact-open" topology.

1. Compact sets of functions. All spaces to be considered in what follows will be assumed Hausdorff.

We introduce now the notation of a $k$-space first defined by Hurewicz.

Definition. A topological space $X$ is called a $k$-space if $S \subset X$ is closed if and only if $S$ intersects every compact subset of $X$ in a compact subset.

The $k$-spaces are an important class in that they are exactly the spaces whose topology is determined once the compact subsets are 1949.

Presented to the Society, December 27, 1949; received by the editors April 23,

1 Numbers in brackets refer to the bibliography at the end of the paper. 
known. Let us verify that $k$-spaces include both locally compact spaces and spaces satisfying the first axiom of countability.

Let $X$ be locally compact and suppose $S \subset X$ intersects every compact set in a compact set. We must show that $S$ is closed, so suppose $x \notin S$. Let $N$ be a neighborhood of $x$ with compact closure $\bar{N}$. Then by assumption $\bar{N} \cap S$ is compact and does not contain $x$, so there is a neighborhood $N^{\prime}$ of $x$ such that $N^{\prime} \cap(\bar{N} \cap S)=\varnothing$. Letting $N^{\prime \prime}=N^{\prime}$ $\cap N$, we get $N^{\prime \prime} \cap S=N^{\prime} \cap N \cap S \subset N^{\prime} \cap \bar{N} \cap S=\varnothing$, so $S$ is closed.

Let $X$ be first countable, and suppose $S \subset X$ intersects every compact set in a compact set. If $x$ is in the closure of $S$, then it is a limit point of a sequence $\left(x_{i}\right)$ of points in $S$, and the set $K$ consisting of the sequence $\left(x_{i}\right)$ and the point $x$ is compact. By assumption $K \cap S$ is compact hence closed, so since each point of $\left(x_{i}\right)$ lies in $K \cap S$, it follows that $x \in K \cap S$ and hence $x \in S$ and $S$ is closed.

We shall now redefine briefly the notions of compact-open (c.o.) and point-open (p.o.) topologies for function spaces.

Definition. If $X$ and $Y$ are spaces and $K \subset X$ is compact and $U$ is open in $Y$, we denote by $[K, U]$ the set of all continuous functions from $X$ to $Y$ such that $f(K) \subset U$. We denote by $Y^{X}$ the space of all continuous functions from $X$ to $Y$ in which the sets $[K, U]$ form a subbase (the compact-open topology).

If $x \in X$ and $U$ is open in $Y$, we denote by $|x, U|$ the set of all functions (not necessarily continuous) from $X$ to $Y$ such that $f(x) \in U$ and we denote by $\left|Y^{x}\right|$ the space of all functions from $X$ to $Y$ in which the sets $|x, U|$ form a subbase (point-open topology).

Some further notation will be needed.

If $F \subset Y^{X}$ and $S \subset X$ and $T \subset Y$ we define,

$F(S)=\bigcup_{f \in F} f(S), \quad \cup F^{-1}(T)=\bigcup_{f \in F} f^{-1}(T), \quad \cap F^{-1}(T)=\bigcap_{f \in F} f^{-1}(T)$.

THEOREM 1. If $X$ is a $k$-space and $Y$ is regular, then a set of functions $F \subset Y^{\mathbf{X}}$ is compact if and only if;

(1) $F$ is closed in $Y^{\mathbf{X}}$;

(2) $F(x)$ is compact for every $x \in X$;

(3) If $Q$ is closed in $F$ and $C$ is closed in $Y$, then $U Q^{-1}(C)$ is closed in $X$.

The condition (3) can be stated in the clearly equivalent form.

$\left(^{\prime}\right)$ If $Q$ is closed in $F$ and $U$ is open in $Y$ then $\cap Q^{-1}(U)$ is open in $X$. (This condition represents a sort of simultaneous continuity of the functions $Q$, requiring that the inverse image of an open set be open in this generalized sense.)

Proof. We first show the necessity of (1), (2), and (3). If $F$ is 
compact, then (1) is satisfied since $Y^{x}$ is Hausdorff [2]. To prove (2), define for each $x \in X, \phi_{x}$ from $Y^{X}$ to $Y$ by $\phi_{x}(f)=f(x)$. Then $\phi_{x}$ is continuous, for if $U$ is open in $Y$ and $\phi_{x}(f) \in U$, we let $N(f)=[x, U]$. $N(f)$ is open by definition of the topology in $Y^{\mathbf{x}}$, and clearly $\phi_{x}(N(x))$ $\subset U$. Therefore $\phi_{x}$ is continuous and hence if $F$ is compact, so is $\phi_{x}(F)=F(x)$.

Condition (3) is the one which replaces equicontinuity and is less trivial to prove. We observe first that if $Q$ is closed in $F$, then it is compact, so it suffices to demonstrate (3) for compact sets of functions, hence for $F$ itself.

Let $C$ be closed in $Y$ and set $E=U F^{-1}(C)$. To show that $E$ is closed, we must show that for any compact set $K, K \cap E=S$ is compact or, what is equivalent, closed. We shall show that if $x_{0} \notin S$, then $x_{0} \notin \bar{S}$. There are two cases.

Case $1, x_{0} \notin K$. Then there is obviously a neighborhood of $x_{0}$ which does not intersect $K$, hence does not intersect $S$, so $x_{0} \in \bar{S}$.

Case 2, $x_{0} \in K$. Then since $x_{0} \notin S$, we have $x_{0} \notin E$, so $f\left(x_{0}\right)$ is in the complement of $C=U$ for all $f \in F$. Since $Y$ is regular we can chose for each $f \in F$ a neighborhood $N_{f}$ of $x_{0}$ such that $f\left(\bar{N}_{f}\right) \subset U$. Now define $K_{f}=\bar{N}_{f} \cap K$. This is a compact set. Finally we define for each $f \in F, W_{f}=\left[K_{f}, U\right]$. These sets are open and cover $F$, so by compactness we can find functions $f_{1}, \cdots, f_{n}$ such that the corresponding $W_{f_{i}}$ cover $F$. Thus for every $f \in F$ there is an $i$ such that $f\left(K_{f_{i}}\right) \subset U$, and therefore if $K^{*}=\bigcap_{i} K_{f_{i}}$, we have $f\left(K^{*}\right) \subset U$ for all $f \in F$ so $K^{*} \cap E=\varnothing$. Now let $N^{*}=\bigcap_{i} N_{f_{i}}$. We show that $N^{*} \cap S$ is empty, for $N^{*} \cap S \subset N^{*} \cap K \subset K^{*}$, so $\left(N^{*} \cap S\right) \cap E=\varnothing$, and since $S \subset E, N^{*} \cap S$ $=\varnothing$. Therefore $S$ is closed and hence compact as it lies in $K$, so since $X$ is a $k$-space we conclude that $E$ is closed.

The proof of sufficiency is quite similar to the proof given by Myers for the equicontinuous case. A preliminary result is needed.

LeммA. If a family $F$ in $Y^{\mathbf{X}}$ satisfies condition (3) of the theorem, then the compact-open and point-open topologies are equivalent on $F$.

Proof. It is clear that every open set in $\left|Y^{\boldsymbol{x}}\right|$ is also open in $Y^{\mathbb{x}}$, giving continuity in one direction. It remains to show that the sets $[K, U] \cap F$ are also open in the point-open topology. Let $f \in F$ map $K$ compact into $U$ open in $Y$. Then for each $x \in K$, choose $V_{x}$ open so that $f(x) \in V_{x} \subset \bar{V}_{x} \subset U$, which is possible since $Y$ is regular. Since $f(K)$ is compact, we choose $x_{1}, \cdots, x_{n}$ such that $U_{i} V_{x_{i}} \supset f(K)$ and let $V=\bigcup_{i} V_{x_{i}}$. Then $f(K) \subset V \subset \bar{V} \subset U$.

Now for each $x \in K$, define $W_{x}=\left\{f^{\prime} \mid f^{\prime} \in F\right.$ and $\left.f^{\prime}(x) \in \bar{V}\right\} . W_{x}$ is closed and nonempty, containing $f$. Therefore it follows from con- 
dition $\left(3^{\prime}\right)$ that $\cap W_{x}^{-1}(U)$ is an open neighborhood of $x$, denoted by $N_{x}$. The neighborhoods $N_{x}$ cover $K$ and hence we can find $x_{1}^{\prime}, \ldots$, $x_{m}^{\prime}$ in $K$ such that $\bigcap_{i} N_{x_{i}^{\prime}} \supset K$.

Now $f \in \bigcap_{i}\left|x_{i}^{\prime}, V\right|$. We shall show $\bigcap_{i}\left|x_{i}^{\prime}, V\right| \subset[K, U]$, for suppose $f^{\prime}\left(x_{i}^{\prime}\right) \in V$ for all $i$. Then for any $x \in K$ there is an $i$ such that $x \in N_{x_{i}}$, hence $x \in \cap W_{x_{i}}^{-1}(U)$ and therefore $g(x) \in U$ for all $g \in F$ such that $g\left(x_{i}\right) \in \bar{V}$. But since $f^{\prime}\left(x_{i}\right) \in V$, it follows that $f^{\prime}(x) \in U$, hence $f^{\prime}(K)$ $\subset U$, completing the proof.

We now obtain the sufficiency of the conditions of Theorem 1 without requiring that $X$ be a $k$-space. Let $F \subset Y^{x}$ satisfy (1), (2), and (3). Since $F$ satisfies (3), it may be considered as imbedded topologically in $\left|Y^{x}\right|$ by the lemma, and we shall denote its image by $F^{\prime}$. Now $\left|Y^{x}\right|$ can be considered as the topological product of $Y$ with itself $X$ times, and since $F(x)$ is compact for each $x$, we get $Q=\prod_{x \in x} F(x)$ is compact, and since $F^{\prime} \subset Q \subset\left|Y^{x}\right|$, it suffices to prove that $F^{\prime}$ is closed in $\left|Y^{x}\right|$.

Suppose then $g^{\prime} \in \bar{F}^{\prime}$. The theorem will be proved if we can show $g^{\prime}$ continuous, for the set $F \cup_{g}$ in $Y^{\boldsymbol{x}}$ will also satisfy condition (3) and will be homeomorphic to $F^{\prime} \cup g^{\prime}$ so that $g \in \bar{F}$. But since $F$ is closed in $Y^{x}$, we have $g \in F$ or $g^{\prime} \in F^{\prime}$, so $F^{\prime}$ is closed, hence compact.

We show $g^{\prime}$ is continuous. Let $x_{0}$ be in $X$ and $g\left(x_{0}\right)$ in $U$ open in $Y$. Choose $V$ open in $Y$ such that $g\left(x_{0}\right) \in V \subset \bar{V} \subset U$ and let $T=\{f \mid f \in F$ and $\left.f\left(x_{0}\right) \in \bar{V}\right\} . T$ is closed in $F$ and nonempty, since $g^{\prime} \in Q$ so that $g^{\prime}\left(x_{0}\right) \in F\left(x_{0}\right)$, and hence for some $f_{0} \in F$ we have $f_{0}\left(x_{0}\right)=g^{\prime}\left(x_{0}\right) \in \bar{V}$. Hence by condition (3), $\cap T^{-1}(V)=N\left(x_{0}\right)$ is open in $X$. We shall show that $g^{\prime}\left(N\left(x_{0}\right)\right) \subset U$. Let $x \in N\left(x_{0}\right)$ and let $W$ be any neighborhood of $g(x)$ in $Y$. Since $g \in \bar{F}^{\prime}$, there exists $f^{\prime} \in F$ such that $f^{\prime} \in\left|x_{0}, V\right|$ $\cap|x, W|$. But $f^{\prime} \in\left|x_{0}, V\right|$ implies $f^{\prime} \in T$, and hence $f^{\prime}(x) \in V$ since $x \in N\left(x_{0}\right)$. Hence $W \cap V \neq \varnothing$ for any $W \ni g(x)$, which means $g(x) \in \bar{V}$ and therefore $g(x) \in U$ and $g$ is continuous.

Remark. Condition (3) of Theorem 1 is not equivalent to equicontinuity when $Y$ is metric. In fact one easily constructs an equicontinuous family from the unit interval to the reals which does not satisfy (3).

We now derive a simple consequence of Theorem 1 .

THEOREM 2. Let $F$ be a family of continuous functions from $a k$-space $X$ into $Y$ regular. Define $\phi: X \rightarrow Y^{P}$ by $\phi(x)(f)=f(x)$. Then $\phi$ is continuous.

Proof. Let $x_{0}$ be in $X$ and let $[K, U]$ be a subbasic neighborhood of $\phi\left(x_{0}\right)$ in $Y^{F}$, with $K$ compact in $F, U$ open in $Y$. This means $K\left(x_{0}\right) \subset U$ 
or $\phi\left(x_{0}\right) \in \mathrm{U}_{K^{-1}}(U)$. But by Theorem $1, N\left(x_{0}\right)=\cap K^{-1}(U)$ is open in $X$ and, since $\phi\left(N\left(x_{0}\right)\right) \subset[K, U], \phi$ is continuous.

2. Application to function rings. Let $R$ denote the real numbers, $X$ a topological space, and $R(x)$ the ring of all continuous functions from $X$ into $R$ in the compact-open topology. A sub-algebra $A(X)$ $\subset R(X)$ will be called separating over $X$ if for $x \neq x^{\prime} \in X$, there exists $f \in A(X)$ such that $f(x)=0, f\left(x^{\prime}\right) \neq 0$.

We define $H$ to be the set of all continuous homomorphisms from $A(X)$ onto $R$ and we define the natural mapping, $\phi$, from $X$ into $H$ as follows: $\phi(x)(f)=f(x)$ for all $f \in A(X)$. To justify this definition one must show that $\phi(x)$ is actually a continuous homomorphism of $A(X)$ onto $R$, which fact, however, is immediately verified.

Lemma. If $A(X)$ is separating, then $\phi$ is one-to-one and onto.

Proof. The one-to-one property follows from the fact that $A$ is separating. Namely $\phi(x)=\phi\left(x^{\prime}\right)$ implies $\phi(x)(f)=\phi\left(x^{\prime}\right)(f)$ for all $f \in A(X)$, or $f(x)=f\left(x^{\prime}\right)$ and hence $x=x^{\prime}$.

To show that $\phi$ maps onto $H$, let $h$ be a continuous homomorphism from $A(X)$ onto $R$ and let $M$ be the closed maximal ideal which is its kernel. We shall show that there exists $x_{0} \in X$ such that $f\left(x_{0}\right)=0$ for all $f \in M$, for suppose this is false. Then $M$ is again a separating algebra on $X$, for given $x \neq x^{\prime} \in X$ choose $f \in M$ such that $f\left(x^{\prime}\right) \neq 0$, and $g \in A(X)$ such that $g(x)=0, g\left(x^{\prime}\right) \neq 0$. Then if $k=f g \in M$ we have $k(x)=0, k\left(x^{\prime}\right) \neq 0$. Now since $M$ is separating, we can apply the Stone-Weierstrass approximation theorem [3] which states that a separating algebra of continuous functions is dense in $R(x)$ in the c.o. topology. But since $M$ is closed, this would mean $M=A(x)$, contrary to the fact that $h$ maps onto $R$. The contradiction shows that every function in $M$ vanishes on some point $x_{0} \in X$. Since $M$ is maximal, it follows that $M$ consists of all $f \in A(X)$ such that $f\left(x_{0}\right)=0$. Thus $h$ has the same kernel as the homomorphism $\phi\left(x_{0}\right)$, which means they differ by an automorphism of $R$, and since the only such automorphism is the identity we conclude that $h=\phi\left(x_{0}\right)$.

The above shows that $X$ and $H$ are in a natural one-to-one correspondence. The question then occurs as to whether one can make $\phi$ into a homeomorphism by giving $H$ a function space topology. We consider the case where the algebra $A(X)$ is completely regular, which means, for any $C$ closed in $X$ and $x \notin C$, there exists $f \in A(X)$ such that $f(x) \notin \overline{f(C)}$. For this case it is known and easily verified that $\phi$ is a homeomorphism when $H$ is given the p.o. topology. First, 
$\phi$ is continuous for if $|f, U|$ is subbasic in $H$, then $\phi^{-1}(|h, U|)$ $=\phi^{-1}\{h \mid h(f) \in U\}=\{x \mid f(x) \in U\}=f^{-1}(U)$, which is open since $f$ is continuous. Also $\phi^{-1}$ is continuous, for let $C$ be closed in $X$ and suppose $h \notin \phi(C)$. If $x=\phi^{-1}(h)$, then by complete regularity there exists $f \in A(X)$ such that $f(\dot{x}) \oplus \overline{f(C)}$ and if we let $U$ be the complement of $\overline{f(C)}$ it follows that $|f, U|$ is a subbasic neighborhood of $h$ which does not intersect $\phi(C)$, so $\phi(C)$ is closed.

As an application of the results of $\$ 1$ we prove:

THEOREM 3. If $X$ is a $k$-space and $A(X)$ is completely regular, then if $H$ is given the c.o. topology, $\phi$ is a homeomorphism.

Proof. The continuity of $\phi$ is precisely the statement of Theorem 2. The fact that $\phi^{-1}$ is continuous is seen since we have observed that $\phi^{-1}$ was continuous in the p.o. topology and a fortiori continuous in the stronger c.o. topology.

We remark that the above theorem shows that p.o. and c.o. topology are equivalent on $H$.

\section{BibliogRAPHY}

1. S. B. Myers, Equicontinuous sets of mappings, Ann. of Math. vol. 47 (1946) pp. 496-502.

2. R. F. Arens, $A$ topology for spaces of transformations, Ann. of Math. vol. 47 (1946) pp. 476-495.

3. M. H. Stone, Applications of the theory of boolean rings to general topology, Trans. Amer. Math. Soc. vol. 41 (1937) pp. 375-481.

Princeton University 